\title{
An analytic study for the effect of antenna height on line-of-sight VHF/UHF communications coverage distance applied to Baghdad city
}

\author{
Firas Abdul Elah Abdul Kareem and Rasha Hassan Abbas \\ Computer Technology Engineering Department, Al-Mansour University College, Iraq
}

\begin{abstract}
A radio system conveys information from transmitter to the receiver. The information can be sent by any antenna that transforms the RF signal electromagnetically through free space medium for electromagnetic wave propagation. Long-distance data communication has been higher in effect through wireless networks. Generally, VHF and UHF radio frequencies are termed as line-of-sight (LOS). In point of fact, the considered horizon for visual LOS is not identical as the horizon for radio wave propagation because of geographic obstacles and the earth curvature limits LOS transmission. This research provides an overview, study, analysis, and implementing simulations with single-antenna and two-antenna systems using MATLAB programming language based on antenna height effect on determining the distance range that can be expected in a radio link. Also, the effects of weather and earth terrain changes effects on coverage distance are also taken in consideration. Baghdad city is considered as the place where the research ideas are applied.
\end{abstract}

Keywords: $\quad$ Omni directional antenna, LOS, optical line of sight, radio line of sight, and
$\mathrm{K}$-factor.

\section{Corresponding Author:}

Firas Abdul Elah Abdul Kareem,

Computer Technology Engineering Department

Al-Mansour University College, Iraq

Baghdad, Iraq.

E-mail: Firas.abdulkareem@muc.edu.iq

\section{Introduction}

Wireless communication stands for the biggest technological contributions to mankind, involving the information transmission over a distance wirelessly. The transmission distance is feasibly ranged from few meters as in TV remote control or thousands of kilometers as in a radio link. Numerous devices have been used for wireless communication as in cordless telephones, GPS entities, mobiles, satellite television as well as wireless computer components [1].

The foremost wireless networks have industrialized within pre-industrial stage in the past. These networks sent information over line-of-sight distances (presently expanded by telescopes) by means of torch signaling, smoke signals, flashing mirrors, semaphore flags and signal flares. A highly structured group of signal combinations has been technologically developed to transfer complex messages with these elementary signals. Observational stations had been constructed on summits and along roads to communicate these messages over huge distances [2].

In the premature days of wireless technology, it has, in general, presumed that lower frequencies have been superior for a long-range communication, and this belief has been true for ground-wave propagation. Radio specialists have been, consequently, referred to the "useless" wavebands of "200 meters and down" from 1.5 
MHz and above. Nevertheless, the specialists in late 1921, found the abilities of high frequencies (HFs) for long-range communication through the ionosphere by means of just simple antennas at (2-30 MHz) frequency range. In terms of line of sight (LOS) distances, very high frequency (VHF) communication from 30 to 300 $\mathrm{MHz}$, and ultra high frequency (UHF) communication from $300 \mathrm{MHz}$ to $1 \mathrm{GHz}$ have been formerly supposed to be restricted. On or about second world war, numerous scattering modes as in meteor and troposphere, have been reliably provided beyond-LOS (BLOS) communication. Currently, various LOS and BLOS infrastructures and radar systems are operated at HF/VHF/UHF. At HF/VHF/UHF, the factors of line length and component outline stand for substantial design matters. Nonetheless, the wavelengths are still great enough in which lumped elements are in general favored to or employed together with transmission lines for filtering in addition to matching [3].

VHF stands for an International Telecommunication Union (ITU) termed for radio waves within 30-300 MHz frequency range based on applied wavelengths within 1-10 meter [4]. VHF propagation ideally features square measure for short-distance terrestrial communication with a diversity slightly farther than LOS from the transmitter [5]. Typical applications of VHF are TV broadcast, FM broadcast, long vary digital communication with radio modems, Amateur Radio, land mobile stations, marine communications, and air navigation systems and traffic management communications [5].

UHF stands for an ITU term for radio frequencies within $300 \mathrm{MHz}$ to $3 \mathrm{GHz}$ based on ranged wavelengths from $1 \mathrm{~m}$ to $1 \mathrm{dm}$. This band involves positioning, navigation, mobile communication, and meteorological satellites [4].

UHF represents the highly characteristically used frequency bands for TV signals transmission. Trendy mobile phones conjointly send and receive under radio frequency spectrum. Radio frequency has been widely employed by public service agencies for two-way radio communication, characteristically victimization narrowband FM. Nevertheless, digital services square measure on the intensification. Narrowband radio modems are based on radio frequency besides VHF frequencies and can be used in management of power distribution networks and dissimilar Supervisory Control and Data Acquisition (SCADA) and automation applications). There has in history been little or no radio broadcasting throughout this band up to date. On the other hand, world positioning system conjointly employs radio frequency [5].

This paper investigates VHF and UHF bands communications as a result of their important applications (as mentioned in above paragraph), and also due to the fact that their coverage distance is directly and strongly affected by antenna height, weather and earth terrain changes.

\section{Omni Directional Antenna}

Antennas stand for elementary constituents of any electric system. Generally, it is a device employed for radiating or receiving electromagnetic energy of radio signal from/into space [6]. The IEEE Standard (IEEE Std 145-1983), describes the antenna as means for radiating or receiving radio waves. It is any device that transforms electronic signals to electromagnetic waves (and vice versa) efficiently with smallest loss of signals. Accordingly, antennas have an imperative role in discovering the features of the system that antennas are employed. They are used in diverse systems in diverse forms. Specifically, in several systems, their operational characteristic are designed around the directional features of the antennas or the antennas are used simply in some others systems to radiate electromagnetic energy in an omni-directional or in numerous systems for point-to-point communication purpose in which augmented gain and lessened wave interference are requested [7]. Omni-directional antennas have been universally used in wireless networks [6].

They spread electromagnetic energy uniformly in all directions as in Figure (1). They typically radiate strong waves in two dimensions, and they are effective for irradiating areas in which the location of other wireless stations will vary with time. A good example of omni-directional antennas are the ones used by radio stations. These antennas are very effectual for radio signal transmission since the receiver may not be stationary. Consequently, listeners get the signal regardless of the direction [8]. 


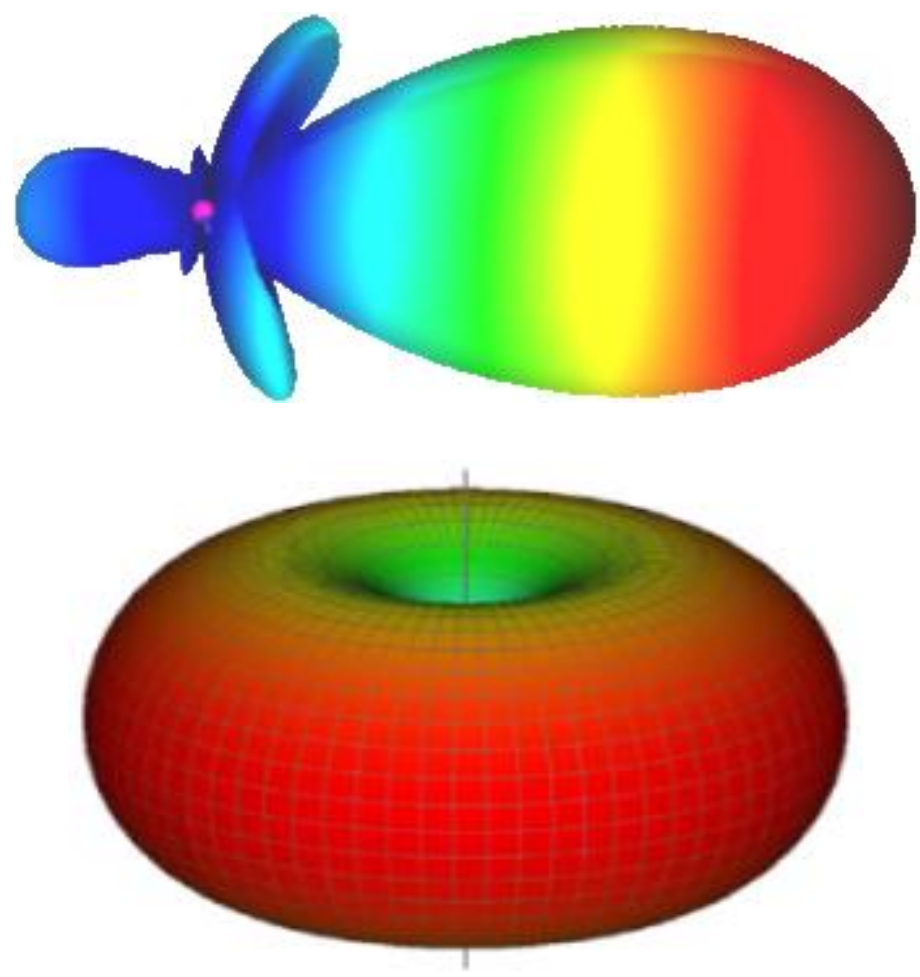

Figure 1. Omni-directional antenna versus directional antenna radiation patterns

Omni-directional antennas radiate or receive equally in all directions. As it does not support a specific direction, it is also known as non-directional. During transmission, the radiated signal has similar strength in every direction, which is helpful in broadcasting a signal to all stations of that area. While receiving, all signals are received equally [8].

\section{Line-of-Sight Propagation}

Direct waves move in a straight line, being feebler as distance upsurges. They can be bent, or refracted, by the atmosphere that spreads their useful range to some extent away from the horizon. Sending and receiving antennas should be have the ability to "see" each other for communications to have effect. Therefore, antenna height is critical in establishing range. Accordingly, direct waves are occasionally identified as LOS waves. This is the prime mode of propagation for VHF along with UHF radio waves [9].

Frequencies in the VHF and UHF bands typically penetrate the ionosphere and speed out into space. Namely, the reflection off the ionosphere can't be adopted to dependably lengthen wireless ranges of these frequencies. For the most part, the sending and receiving antennas should have a properly passable path amid them for communication to happen based on line-of-sight (LOS). LOS stands for radio signal propagation in straight line from the sender to a receiver without refraction. In general, it extends to the visible horizon [9]. Shortrange radio communications for LOS communications can occur at all radio frequencies, nevertheless that task is most frequently specified in the VHF and UHF radio bands [9].

Radio waves can be refracted, or bent, somewhat as roaming from one medium to another. Refraction is resultant from a change in the velocity of a wave as it crosses the boundary within one propagating medium and another. If this transition is created at an angle, one part of the wave front delays or accelerates before the other. As a consequence, bending the wave is occurred to some extent as presented in Figure (2). The lines signify the crests of a moving wave front and the distance among them is the wavelength. The wave direction alters since one end of the wave delays before the other as it crosses the boundary between the two media. The wavelength is concurrently reduced, but the wave frequency (crest number that passes a certain point in a 
given unit of time) is constant. The quantity of bending rises with the ratio of the refractive indices of the dual mediums [10].

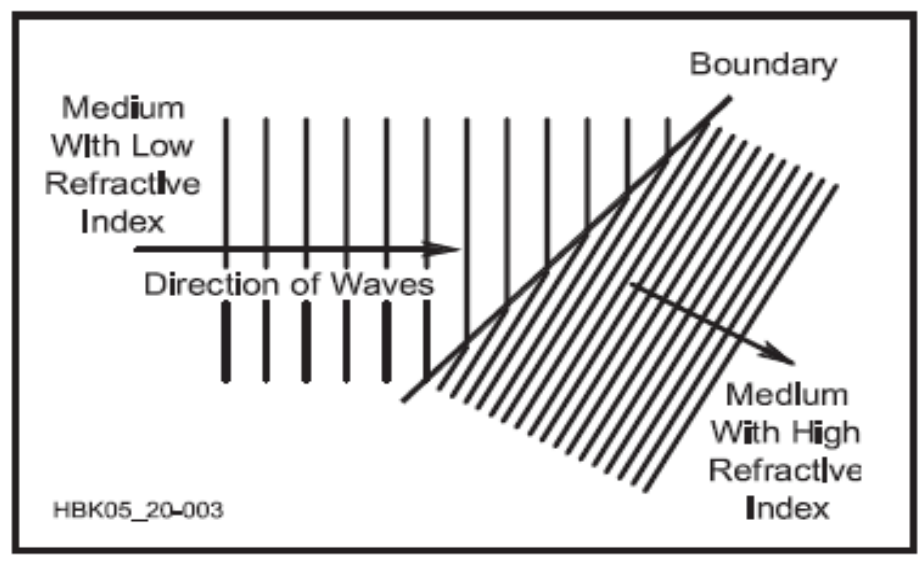

Figure 2. Refracted radio waves as they pass at an angle between dissimilar media

Refractive index ( $\mathrm{K}$ factor) stands for a scaling factor (actually presumed as a constant for a specific path) that measures curvature of a radiated ray pathway. Typical radio links, which are designated as LOS, erroneously put forward that in effect communications are restricted by the optical horizon (i.e., $\mathrm{K}=1$ ). Frequently, radio links are not restricted to LOS propagation. Indeed, we frequently can accomplish communications beyond the optical horizon by a rate of $15 \%$ (i.e., $\mathrm{K}=1.33$ ) as depicted in Figure (3) [11]. If the factor $\mathrm{K}$ is bigger than 1 , the ray beam is bent in the direction of the earth and the radio horizon is bigger than the optical horizon. If $\mathrm{K}$ is less than 1, the radio horizon is lower than the optical horizon. For typical system planning purposes, $\mathrm{K}=\frac{4}{3}$ can be used. Nevertheless, for particular path engineering, $\mathrm{K}$ should be chosen intently [12].

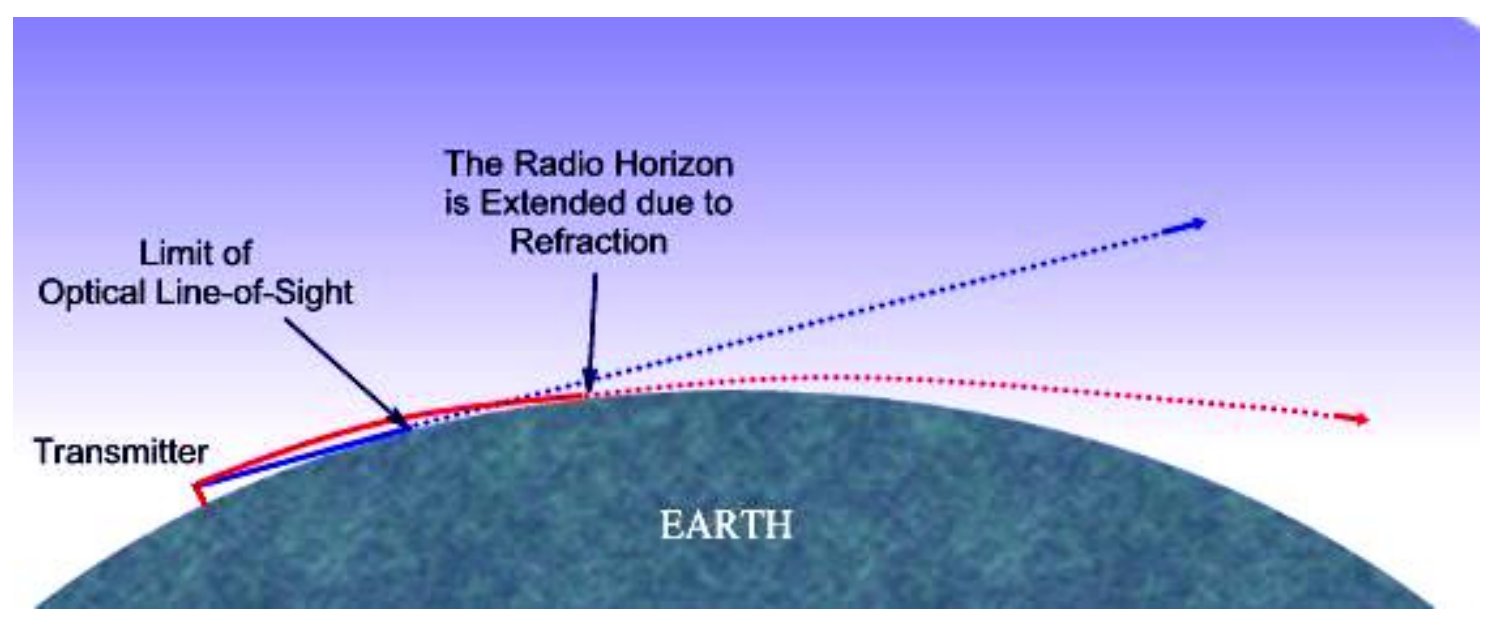

Figure 3. Optical line-of-sight versus radio line-of-sight

For optical and radio LOS with no intervening obstacles, the optical LOS can be stated as [13]:

$$
\mathrm{d}=3.57 \sqrt{\mathrm{h}}
$$

Where $\mathrm{d}$ is the distance between an antenna and the horizon in kilometers and $\mathrm{h}$ is the antenna height in meters. The effective, or radio, LOS to the horizon is stated as [13]:

$$
\mathrm{d}=3.57 \sqrt{\mathrm{Kh}}
$$

where $K$ is an tuning factor to account for the refraction. A worthy rule of thumb is $K=4 / 3$. In consequence, the extreme distance between two antennas for LOS propagation is: 


$$
\mathrm{d}=3.57\left(\sqrt{\mathrm{Kh}_{1}}+\sqrt{\mathrm{Kh}_{2}}\right)
$$

where $h_{1}$ and $h_{2}$ stand for the heights of the two antennas [13].

\section{Results and Discussion:}

\section{a. The Effect of Antenna Height on Coverage Distance:}

To measure the effect of antenna height on coverage distance according to equations (1) (2), and (3), we assume that propagation is perfect which means that $\mathrm{K}$-factor is $4 / 3$ (weather is standard atmosphere, and terrain is temperate zone with no fog). We assume that the first antenna (base station antenna) of two-antenna system is installed on $3 \mathrm{~m}$ one-floor building with antenna mast height of $2 \mathrm{~m}$.

Figure (4) shows the relationship between the height of the antenna and the distance between an antenna and the horizon in kilometers, when using the optical and radio horizon formulas for single antenna system and when installing a K-factor on $4 / 3$ and antenna height range up to $100 \mathrm{~m}$. We notice that increasing antenna height increases the optical or radio coverage distance and increasing antenna height not only increases coverage distance, but it reduces the effect of objects on ground on the oscillating electromagnetic radio waves.

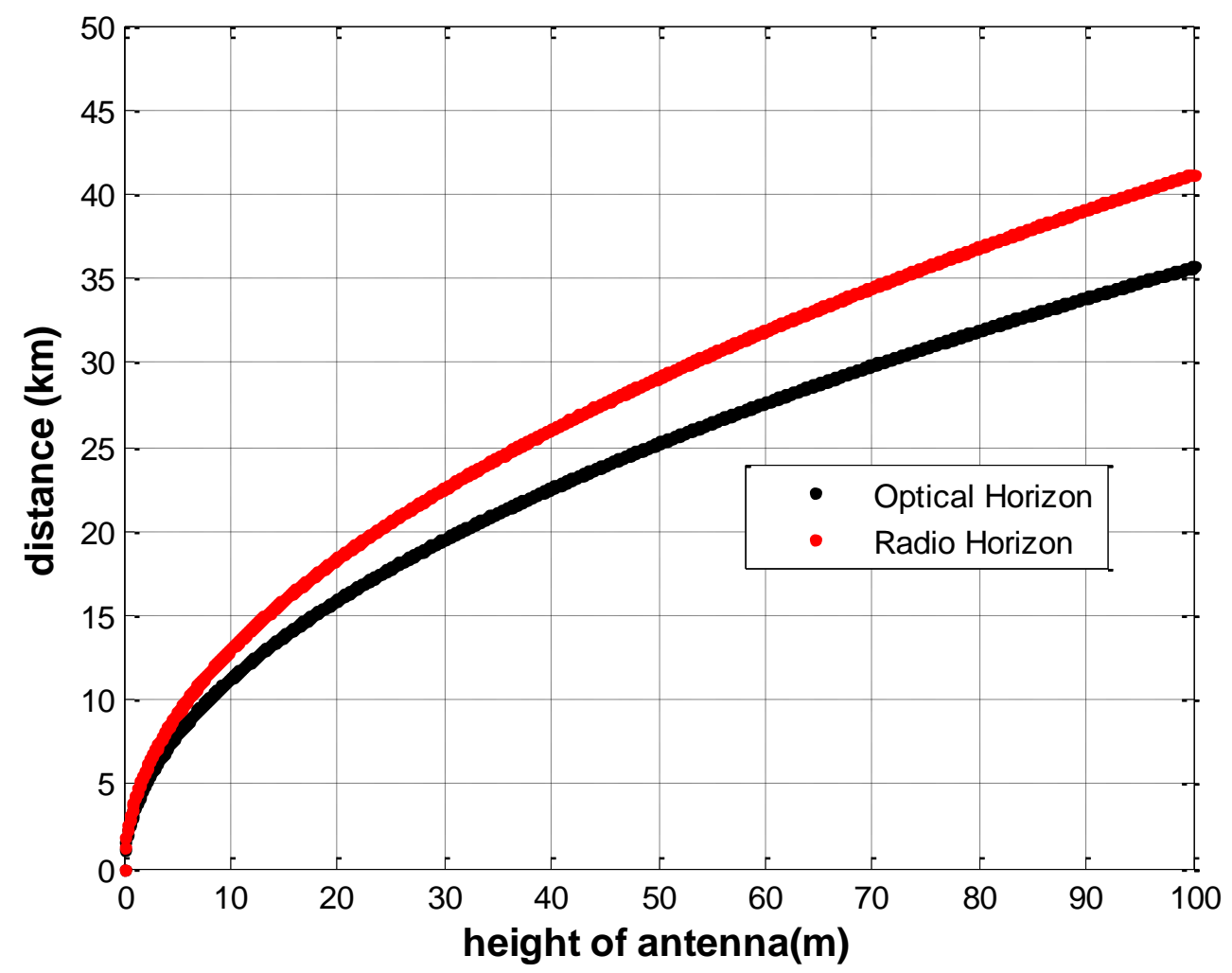

Figure 4. Optical and radio LOS for single antenna system when $\mathrm{K}$-factor=4/3, and antenna height range up to $100 \mathrm{~m}$

Figure (5) shows the relationship between the height of the antenna and the distance between two antennas for radio LOS in kilometres using the radio horizon formulas for two-antenna system and installing the K-factor on $4 / 3$. The height of the first antenna is $5 \mathrm{~m}$ and the second antenna is up to $100 \mathrm{~m}$. We notice that increasing the height of the second antenna in two antenna system increases the coverage distance and can gain more line of sight coverage distance than one-antenna system. 


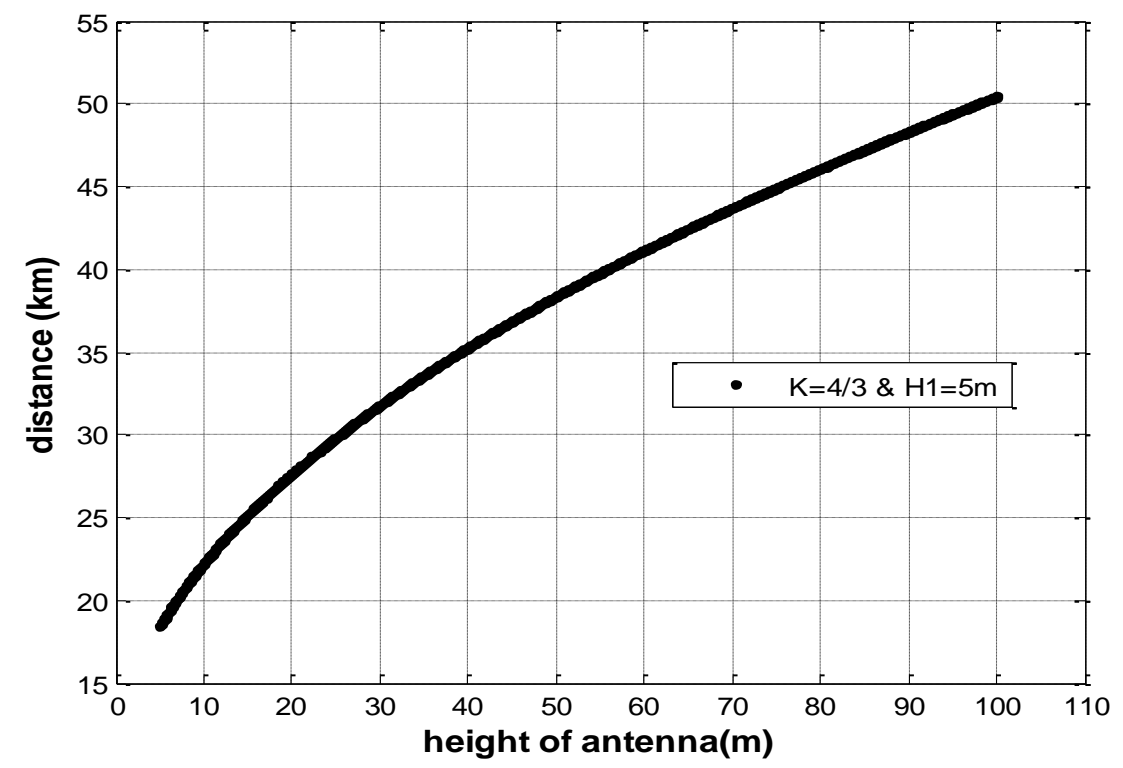

Figure 5. Radio LOS for two-antenna system

\section{b. The Effect of the First Antenna Height in Two-antenna System on the Coverage Distance:}

To measure the effect of the first antenna height on coverage distance according to equation (3), we assume that propagation is perfect which means that $\mathrm{K}$-factor is $4 / 3$ (weather is standard atmosphere, and terrain is temperate zone with no fog). Figure (6) shows the relationship between the height of the second antenna and the distance between two antennas for radio LOS in kilometers when the second antenna is up to $100 \mathrm{~m}$ and the height of the first antenna (h1) is firstly taken $5 \mathrm{~m}$ then it has taken $20 \mathrm{~m}$. We notice that the increase in this height leads to a little bit increase in the coverage distance.

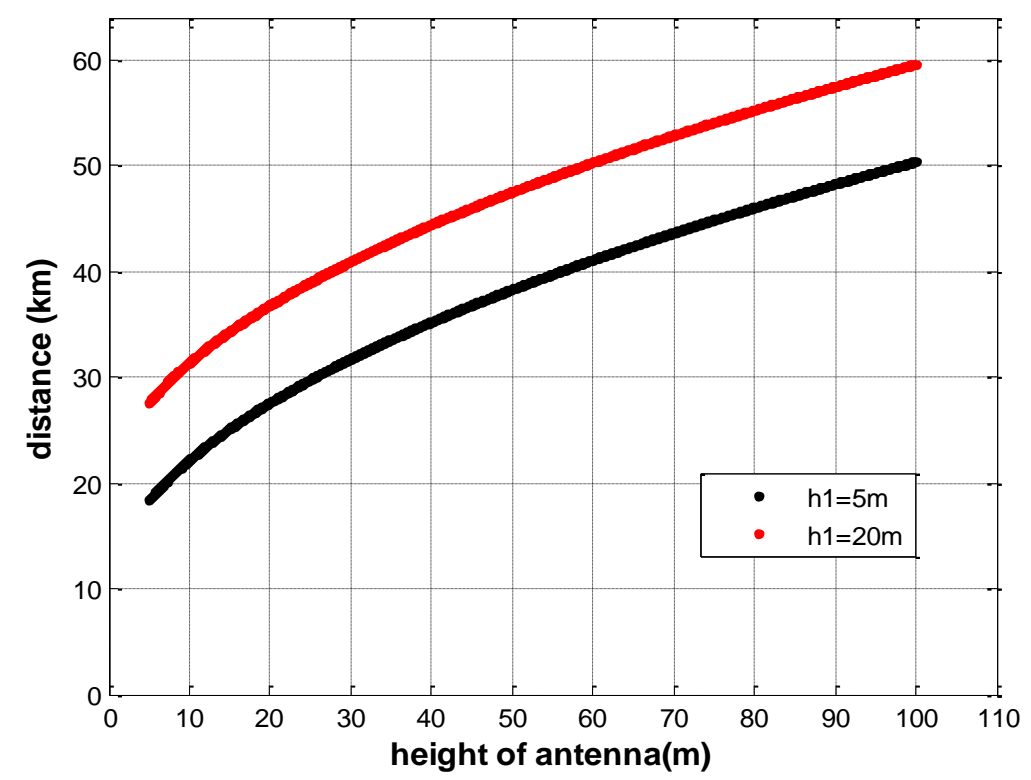

Figure 6. Radio LOS for two-antenna system when $\mathrm{h} 1=5 \mathrm{~m}$ and $20 \mathrm{~m}$

\section{c. The Effect of K-Factor on Coverage Distance:}

To measure the effect of the K-factor on coverage distance according to equations (1), (2), and (3), we assume that the range of the antenna height for single-antenna system is equal to the range of the second antenna height for two-antenna system which varies up to $100 \mathrm{~m}$ and we assume that the first antenna (base station 
antenna) of two-antenna system is installed on $3 \mathrm{~m}$ one-floor building with antenna mast height of $2 \mathrm{~m}$. The followings are some well-known practical cases in which a radio frequency engineer may face:

Propagation is perfect ( $\mathrm{K}$-factor $=4 / 3$ ), weather is standard atmosphere, and terrain is temperate zone with no fog (see Figure (7)).

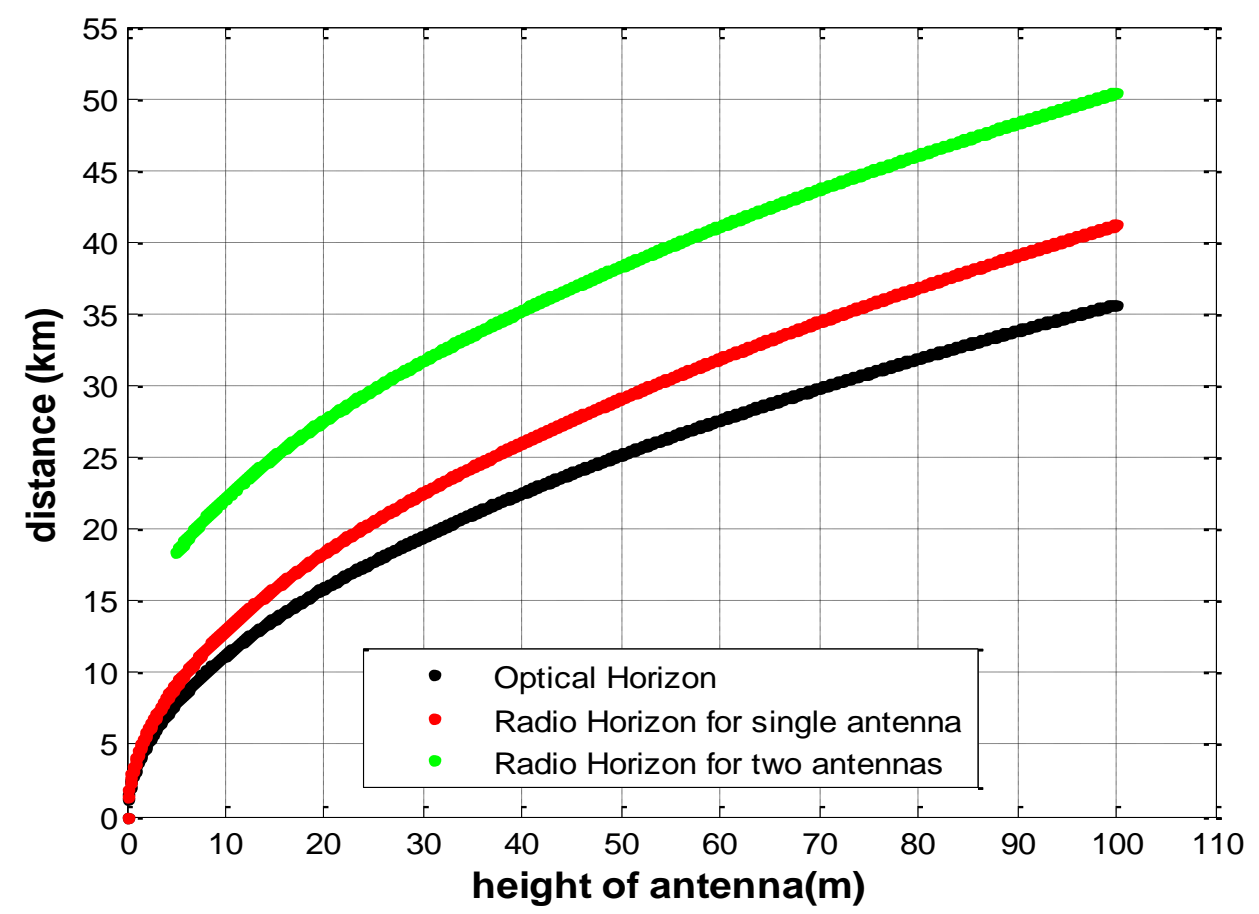

Figure 7. Radio and optical LOS for single-antenna system and radio LOS for two-antenna system when Kfactor $=4 / 3, \mathrm{~h}=\mathrm{h} 2$ which varies up to $100 \mathrm{~m}$ and $\mathrm{h} 1=5 \mathrm{~m}$

Propagation is ideal (K-factor $=1-4 / 3$ ), weather is no surface layers with fog, and terrain is dry, mountainous, and no fog (see Figure (8) when K-factor is 1.2).

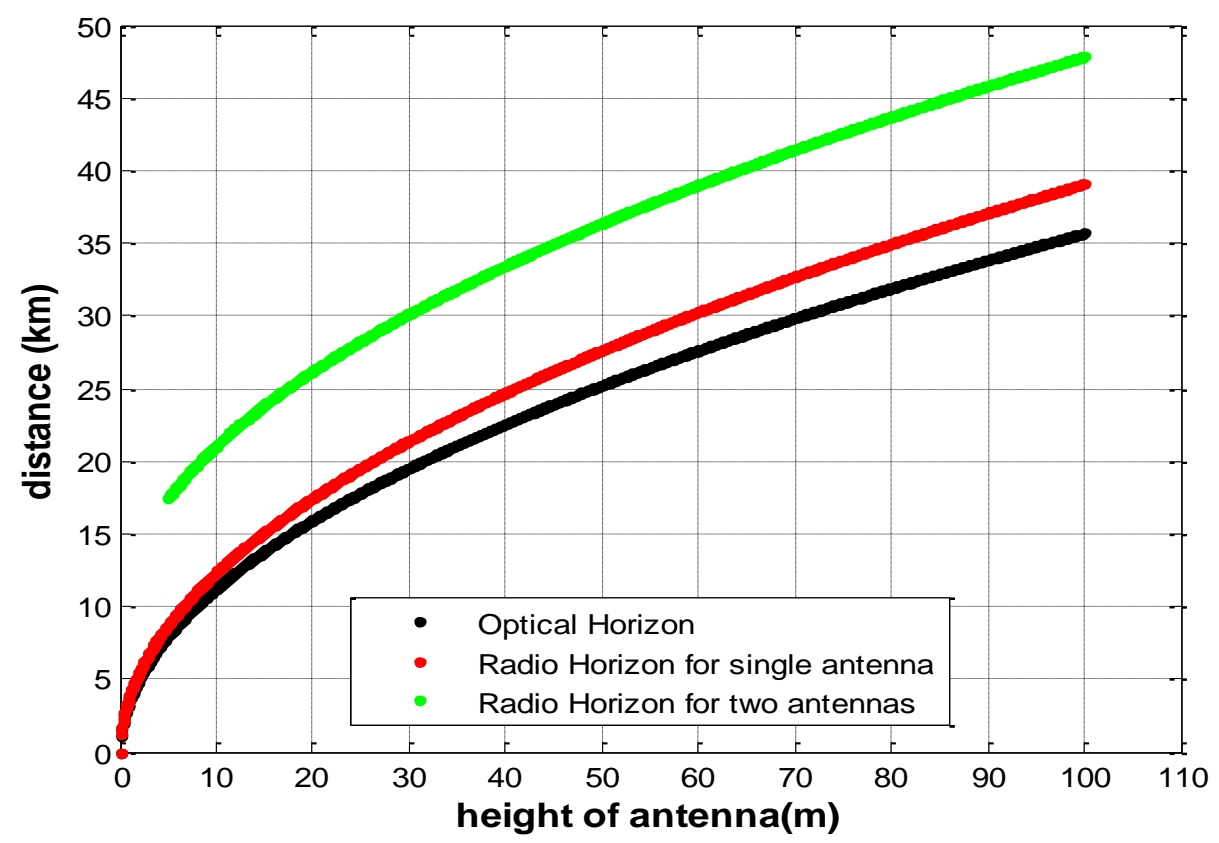

Figure 8. Radio and optical LOS for single-antenna system and radio LOS for two-antenna system when $\mathrm{K}$-factor $=1.2, \mathrm{~h}=\mathrm{h} 2$ which varies up to $100 \mathrm{~m}$ and $\mathrm{h} 1=5 \mathrm{~m}$ 
Propagation is average (K-factor range: $0.66-1.0$ ), weather is substandard with light fog, and terrain is flat, temperate with some fog (see Figure (9) when K-factor is 0.8).

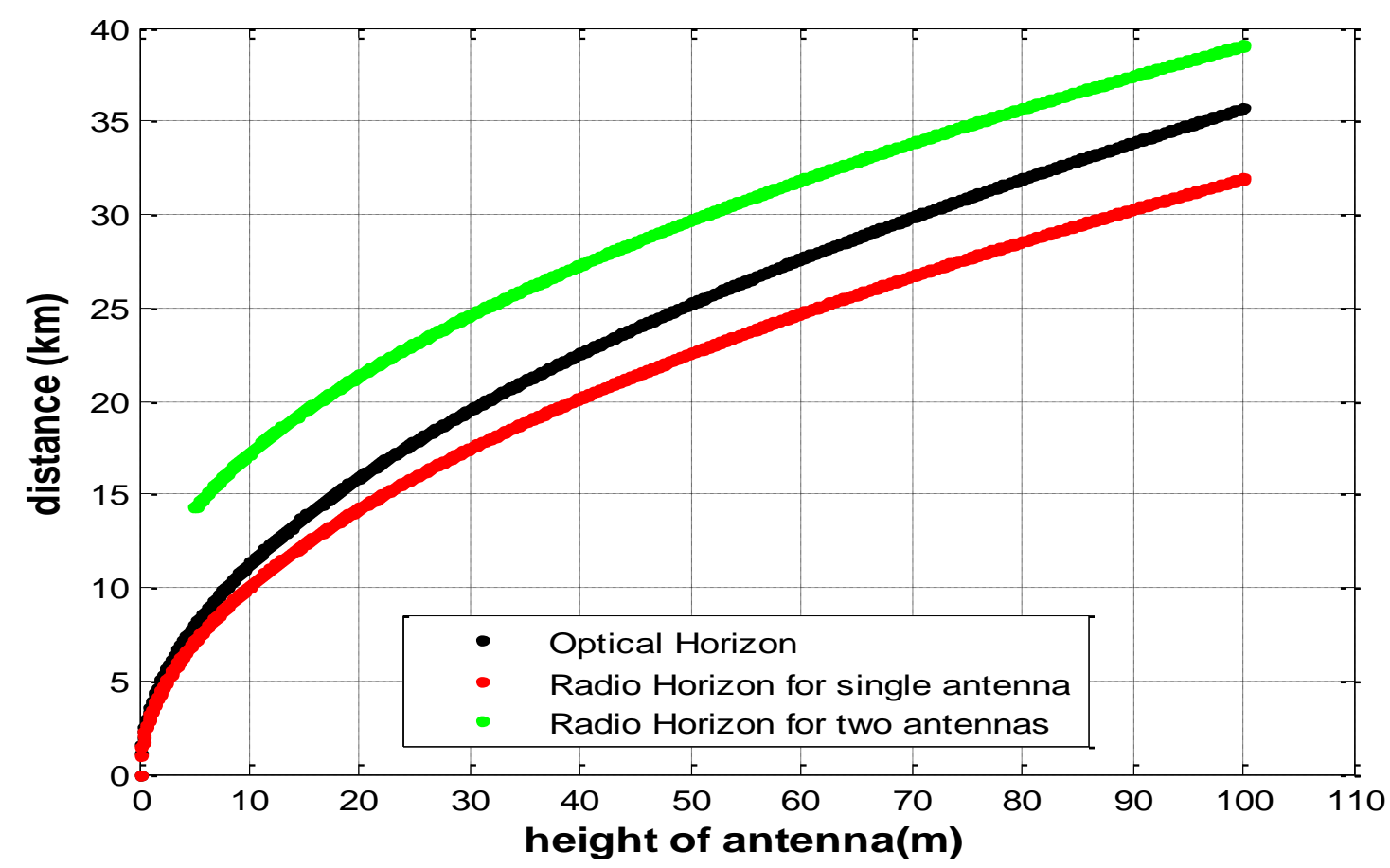

Figure 9. Radio and optical LOS for single-antenna system and radio LOS for two-antenna system when $\mathrm{K}$-factor $=0.8, \mathrm{~h}=\mathrm{h} 2$ which varies up to $100 \mathrm{~m}$ and $\mathrm{h} 1=5 \mathrm{~m}$

Propagation is difficult (K-factor: $0.66-0.5$ ), weather is within surface layers and ground fog, and terrain is coastal (see Figure (10) when K-factor is 0.6).

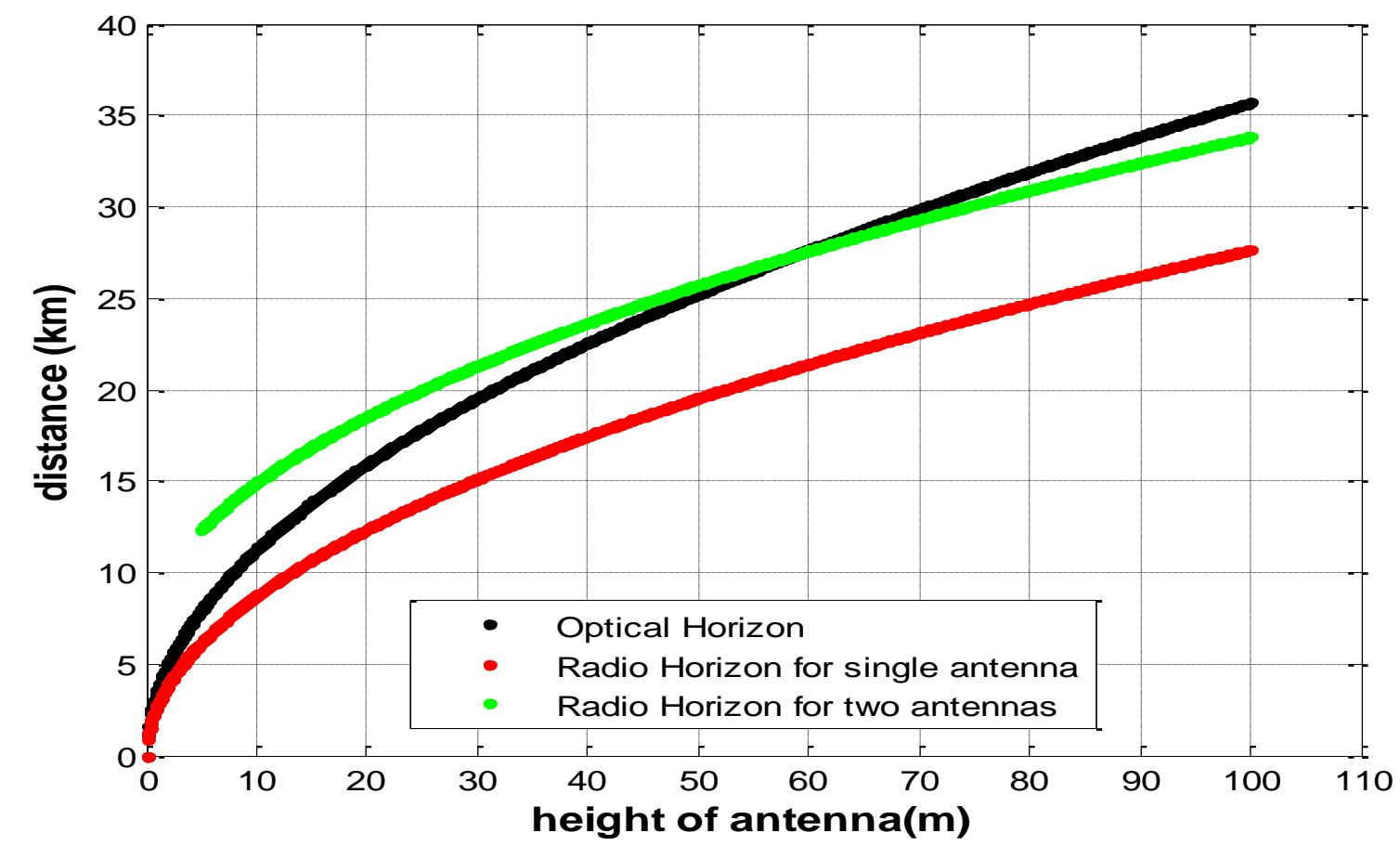

Figure 10. Radio and optical LOS for single-antenna system and radio LOS for two-antenna system when $\mathrm{K}$-factor $=0.6, \mathrm{~h}=\mathrm{h} 2$ which varies up to $100 \mathrm{~m}$ and $\mathrm{h} 1=5 \mathrm{~m}$. 
Propagation is bad (K-factor: $0.5-0.4)$, weather is within fog moisture and over water, and terrain is coastal, water, and tropical (see Figure (11) when K-factor is 0.45).

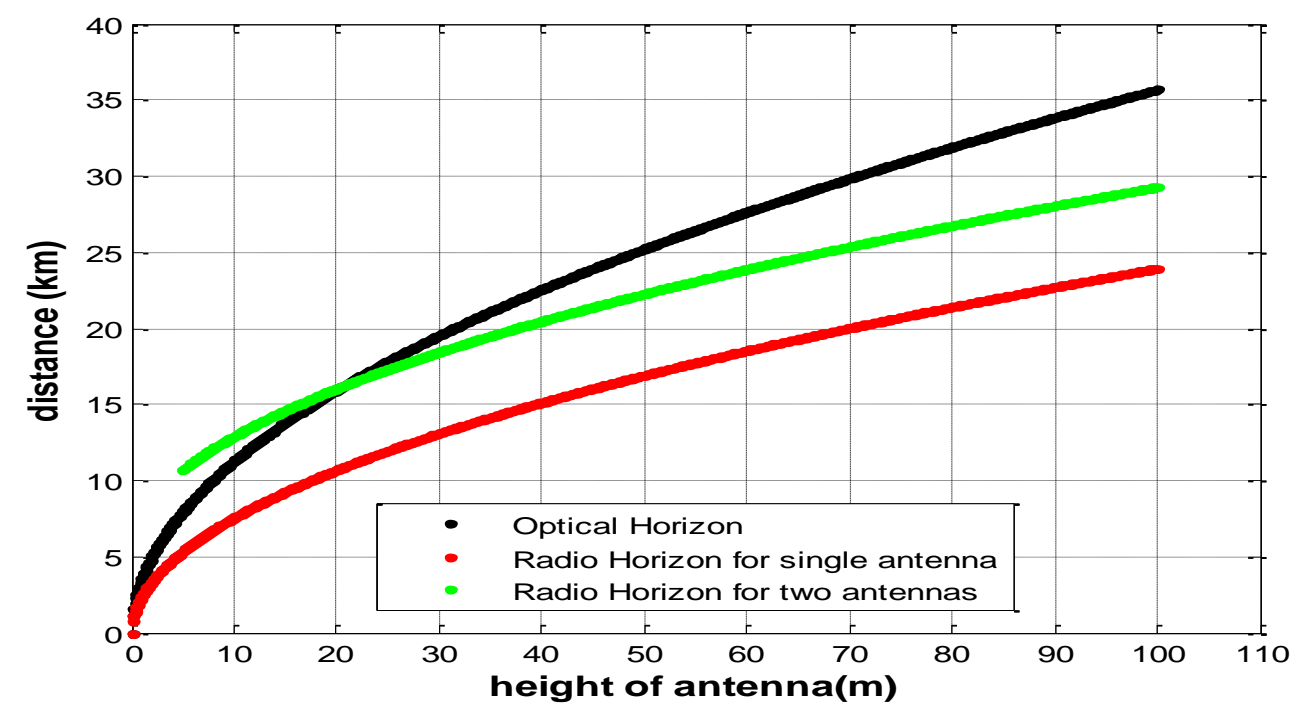

Figure 11. Radio and optical LOS for single-antenna system and radio LOS for two-antenna system when Kfactor $=0.45, \mathrm{~h}=\mathrm{h} 2$ which varies up to $100 \mathrm{~m}$ and $\mathrm{h} 1=5 \mathrm{~m}$

We notice that in one-antenna system, when $\mathrm{K}$-factor is less than 1 , the radio horizon is less than optical horizon. When K-factor is greater than 1, the optical horizon is less than radio horizon. Also, we notice that the coverage distance (radio horizon) of single-antenna system is less than the coverage distance of twoantenna system regardless of the value of K-factor.

\section{d. Simulation of coverage distances using Google Earth map, when installing base station antenna mast at Baghdad centre}

In this part, a Google program is used to access to the Baghdad ground maps for measuring distances between two assumed points through maps and operate geometric shapes on the maps based on accurate measurements of line of sight distances gotten for pre-defined situations.

A base station antenna mast is assumed to be installed on the top highest floor of "Turkish Restaurant" building as maximum available height that could be obtained at Baghdad center to discover the area in Baghdad city that the VHF transmission will cover (see Figure (12)), for different assumed values of K-factor shown in Table (1). The height of the building is about $45 \mathrm{~m}$. The following table shows the colors assigned to coverage areas circles with corresponding K-Factor values.

Table 1. Colors assigned to coverage areas circles in accordance with K-factor values.

\begin{tabular}{|l|l|}
\hline Circle Color & K-Factor Value \\
\hline Black & $4 / 3$ \\
\hline Green & 1.2 \\
\hline Yellow & 0.8 \\
\hline Red & 0.6 \\
\hline White & 0.45 \\
\hline
\end{tabular}

Table 2 illustrates area name in English language according to tags numbered from 1 to 22 (while in the map the names are written in Arabic). 
Table 2. Area name according to tags on the map in Figure 12

\begin{tabular}{|l|l|l|l|}
\hline Tags & Area Name & Tags & Area Name \\
\hline 1 & Baghdad & 12 & Radwaniyah \\
\hline 2 & Al Maghrib & 13 & Abu Ghraib \\
\hline 3 & Sabiat & 14 & Hor Al Basha \\
\hline 4 & Sadr City & 15 & Nahrawan \\
\hline 5 & Tajiat & 16 & Al Wihda \\
\hline 6 & Sabaa Al Bour & 17 & Madain \\
\hline 7 & Al-Ubaidi & 18 & Mahmudiyah \\
\hline 8 & Al-Fahama & 19 & Karma \\
\hline 9 & Hosseinia & 20 & Al-Fallujah \\
\hline 10 & America & 21 & Saqlawia \\
\hline 11 & Hor Rajab & 22 & Amiriyat Fallujah \\
\hline
\end{tabular}

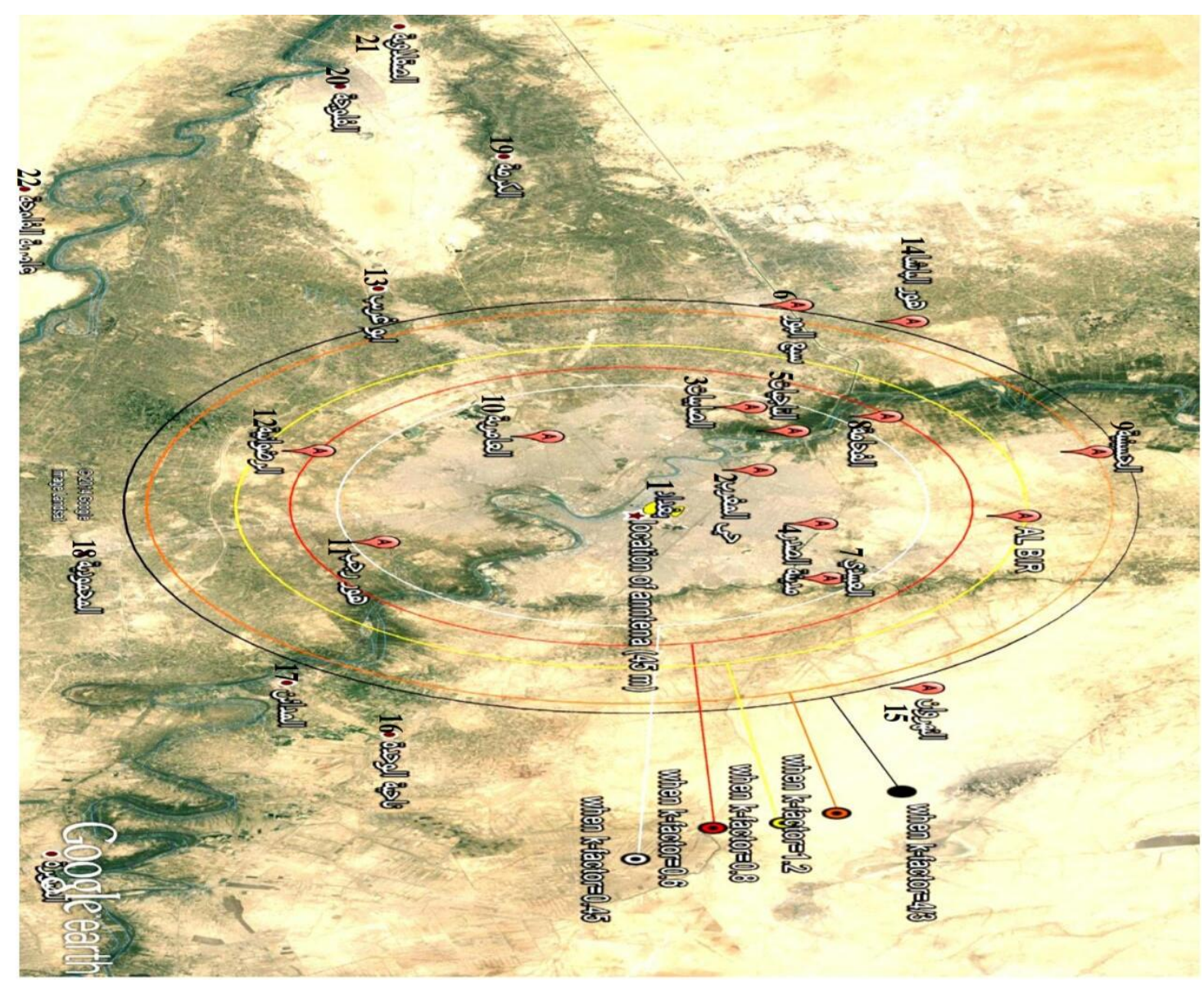

Figure 12. The coverage areas of VHF transmission in Baghdad city for different practical cases values of KFactor

\section{Conclusions}

From the present research, the following conclusions can be drawn as follows:

1. VHF and UHF could be regarded as short distance coverage communication ranges.

2. If at all possible, at antenna installation phase, we should get the antenna above any obstructions by mounting antenna at certain height about ground. 
3. For microwave radio systems, it is not always possible to use long transmission lines between transmitter and antenna, so towers with equipment rooms or cabinets close to the height of the antenna may be required. 4. In single-antenna system, doubling antenna height doesn't automatically double the coverage distance. To double it, the antenna height should be doubled four times.

5. Earth surface terrain has great effect on the gained coverage distance for a certain antenna height.

6. Weather condition has great effect on the gained coverage distance for a certain antenna height. Rain, snow, fog, and any high humidity condition can affect the line of sight case.

7. Increasing antenna height in many situations, gives better coverage distance than increasing transmitted power choice.

8. Increasing antenna height not only increases coverage distance, but it reduces the effect of objects on ground on the oscillating electromagnetic radio waves.

9. Blank communication zones may occur due to terrain, high rise buildings and other structures.

10. It is recommended to perform a radio site survey before installing the equipment; to discover the site practical situation (weather, obstacles, available buildings to choose one for installing antenna mast, temperature at day and night).

11. Two-antenna system can gain more line of sight coverage distance than one-antenna system.

12. Walkie talkie portable radio systems have the lowest line of sight coverage distance, since they have low transmitted power, and are difficult to achieve line of sight case with other radios.

\section{References}

[1] Deepali Y Kirange, and Kalyani N Neve, " Wireless Communication: The Comparative Between Broadcasting, Satellite Communication and Cellular Service", International Journal of Computer Engineering and Technology (IJCET), Volume 4, Issue 5, pp. 31-41, September - October 2013.

[2] Tom Seymour and Ali Shaheen, "History of Wireless Communication", Review of Business Information Systems - Second Quarter, Volume 15, Number 2, pp. 37-42, 2011.

[3] Frederick H. Raab, Robert Caverly, Richard Campbell, Murat Eron, James B. Hecht, Arturo Mediano, Daniel P. Myer, and John L. B. Walker, " HF, VHF, and UHF Systems and Technology", IEEE Transactions on Microwave Theory and Techniques, VOL. 50, NO. 3, pp. 888-899, March 2002.

[4] Indrima Upadhyay and Abhishek Yadav, "Analysis of Deep Space Telecommunication System Parameters, Design Control, Link Analysis and Space Communication Bands", International Journal of Innovative Research in Science, Engineering and Technology (IJIRSET), Vol. 7, Issue 8, pp. 9161-9171, August 2018.

[5] Amit Kumar, Adnan Sherwani, and Akash Singh, " HF, VHF, UHF Technology", International Journal of Innovative Research in Technology (IJIRT), Volume 1, Issue 6, pp. 1316-1317, 2014.

[6] Hong-Ning Dai1, Kam-Wing Ng, Minglu Li, and Min-You Wu3, " An Overview of Using Directional Antennas in Wireless Networks ", International Journal of Communication Systems, 26(4): 413-448, April 2013, Published online in John Wiley \& Sons, Ltd., pp 1-39, 2011.

[7] Pramod Dhande, " Antennas and its Applications ", Armament Research \& Development Establishment, Dr Homi Bhabha Rd, Pashan, Pune-411 021, DRDO Science Spectrum, pp. 66-78, March 2009.

[8] EC-Council, "Wireless Safety", Cengage Learning, 2010.

[9] Harris Corporation, "Radio Communications in the Digital Age (VHF/UHF Technology, Volume 2)", First Printing, June 2000.

[10] H. Ward Silver, "2014 ARRL Handbook for Radio Communications", Ninety-First Edition, The American Radio Relay League, Inc., 2013.

[11] Roger L. Freeman, " Radio System Design for Telecommunications", Third Edition, John Wiley \& Sons, Inc., 2007. 
[12] Roger L. Freeman, " Telecommunication System Engineering", Fourth Edition, John Wiley \& Sons, Inc., 2004.

[13] William Stallings, "Wireless Communications and Networks", Second Edition, Pearson Education Limited, 2014. 